\title{
Multiple reflections and improvement of edge scattering in GRECO RCS prediction code
}

\author{
Juan M. Rius, M.Vall-Ilossera, Lluis Jofre \\ Grup A.M.R., Dpt. Teoria del Senyal i Comunicacions, UPC Apdo. \\ 30002, 08080 Barcelona, \\ TI. $+34-3-4017219$, Fax $+34-3-4017232$
}

\section{Abstract}

GREC0 code for monostatic RCS prediction in real time has been extended by considering multiple reflections between surfaces and improving the edge diffraction coefficients. Multiple reflections are analysed through a very efficient ray-tracing algorithm based on the graphical processing technique. Method of equivalent currents for edge scattering has been improved by Mitzner's and Michaeli's incremental length diffraction coefficients (ILDC).

This communication presents the general features of GRECO code, in particular the advantages of the new graphical processing technique. Emphasis will be placed in the new features of GREC0 still unpublished: the ray-tracing algorithm and the implementation of incremental length diffraction coefficients.

\section{Graphical Electromagnetic Computing (GRECO)}

During the last four years, the development of graphical processing techniques for high-frequency monostatic RCS prediction has given rise to GRECO code (1), (2), (3). Real-time computation is achieved through graphical processing of an image of the target present at the screen of a workstation, using the hardware capabilities of a 3-D graphics accelerator. The two main advantages of the graphical processing approach over classical techniques are:

a) Hardware graphics accelerator removes hidden surfaces and edges: they do not contribute to the surface or line integrals when they are performed by the graphical processing technique.

b) Reduced CPU time and RAM requirements. They are independent of target electrical size and complexity. The main features of GRECO code are the following:

a) The I-DEAS computer aided design package for geometric modelling of solids has been used for modelling target geometry. The target is described as a collection of parametric surfaces, defined with two-dimensional NURBS (non-uniform rational Bsplines). Parametric surfaces require less mass storage memory that the faceting approach, and adjust more accurately to the real target surface.

b) Physical Optics (PO) approach for perfectly conducting surfaces and Impedance Boundary Condition (IBC) + Physical Optics for radar absorbent coatings.

c) Method of Equivalent Currents (MEC) with either Physical Theory of Diffraction (PTD), Mitzner's or Michaeli's Incremental Length Diffraction Coefficients (ILDC) for perfectly conducting edges. A comparative study of the results for the three kinds of ILDC's will be presented in this communication.

d) Double reflection analysis by geometric optics (GO) raytracing for the first reflection and bistatic physical optics for the second. A new and very efficient ray-tracing algorithm has been developed and will be also presented in this communication.

\section{Ray-Tracing}

Double reflections between surfaces are analysed by a hybrid GO-P0 scheme. The G0 reflection at the first surface assumes that specular reflection occurs, according to stationary phase principle. The PO reflection at the second surface ensures that the correct scattered field is obtained when there is no specular reflection to the observer.

For each pixel on the target surface, a reflected ray is traced along the GO specular direction. The impact of the reflected ray with another surface is detected on the screen by following the ray-path and comparing the $\mathrm{z}$ coordinates of the ray with that of the surface at the same $x, y$ location. In case that there is a second reflection, the field scattered to the observer is computed using bistatic PO. Vector formulation of bistatic GO and PO for respectively the first and second reflections allows to obtain the scattering matrix of the target.

This scheme is valid for both planar and curved surfaces. In the former case, the reflected rays are parallel, which is equivalent to a plane wave incident over the second surface. In the later case, the divergence factor due to the curvature of the surface is implicit in the graphical ray-tracing algorithm: the number of rayhits on the second surface is inversely proportional to the divergence of the reflected rays.

\section{Incremental Length Diffraction Coefficients}

It is well known that Ufimtsev's PTD coefficients are accurate for analysing high-frequency monostatic edge diffraction only when the incidence direction is perpendicular to the edge, for which monostatic observation is along the Keller cone (5). This is the case for the main edge contribution to total RCS, so that oblique edges contribution can be usually neglected. For that reason, and due also to their simplicity, PTD coefficients together with Physical Optics constitute the most usual approach to monostatic RCS prediction. Excellent results have been obtained by GREC0 code using a very efficient linear approximation to PTD coefficients (2), (3).

An exception to the above rule is the analysis of stealth aircraft, in which specular surfaces and edges are deliberately avoided. For rigorous analysis of oblique edges it is necessary to use MEC with some kind of incremental length diffraction coefficients, which are correct for observation outside the Keller cone (5). Usually Mitzner's ILDC (5) have been chosen for this purpose in a number of RCS prediction codes but, on the other hand, they are singular for some observation directions (6). This problem has been overcome by Michaeli's ILDC (6), which remove most of the singularities of Mitzner's and, in particular, are finite for all monostatic observations. The main drawback of Michaeli's ILDC is a rather complex formulation, even for the monostatic case, which makes real-time RCS prediction difficult for GRECO code.

\section{Results}

An interesting RCS problem which includes contributions from both double reflections and edge diffraction is the $90^{\circ}$ dihedral proposed at Marseille'92 RCS workshop (4). The geometry of the object is shown in fig. 1. Notice the monostatic sweep along the bisector plane, $\phi=45^{\circ}$, instead of the plane perpendicular to the dihedral faces, $\theta=90^{\circ}$. This unusual sweep makes the contribution of oblique edges more important than that of surface reflection, becoming an excellent test for the accuracy of the different kind of edge diffraction coefficients.

Figure 2 shows the RCS from single and double surface reflections computed by a double $\mathrm{PO}+\mathrm{P} 0$ surface integral compared to the predictions of the $\mathrm{GO}+\mathrm{PO}$ ray-tracing technique implemented in GRECO code. Notice that for grazing incidence the latter is a few $\mathrm{dB}$ under the $\mathrm{PO}+\mathrm{PO}$ result, due to the fact that most $\mathrm{GO}$ rays speculary reflected on a face of the dihedral do not hit the other one, while for these rays PO predicts non-null scattered fields that reach the other face of the dihedral.

The results of GRECO code using the three kinds of edge diffraction coefficients together with the ray-tracing algorithm are shown in fig. 2 (thick line plots). These results have been 


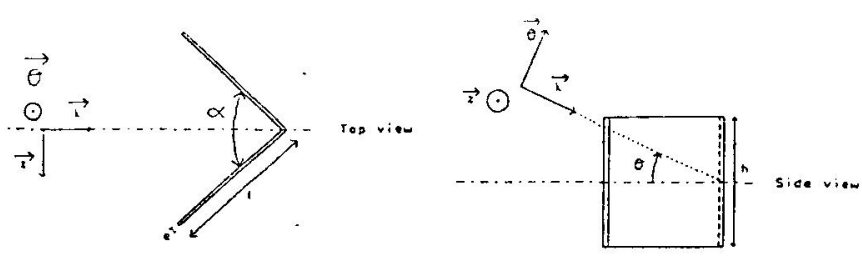

Fig. I: Dihedral, $l=1.5 \lambda, h=2.25 \lambda, \alpha=90^{\circ}$. RCS sweep along bisector plane.

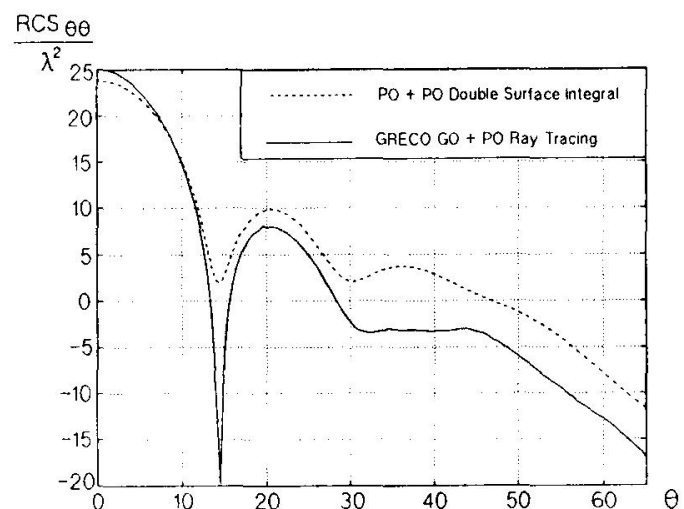

$\frac{\operatorname{RCS}_{22}}{\lambda^{2}}$

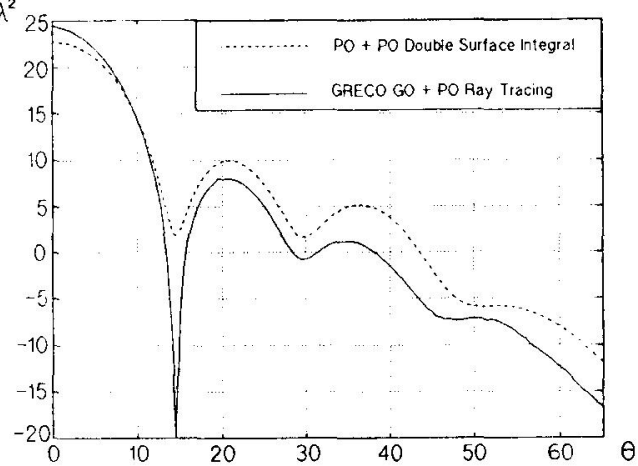

Fig. 2: Contribution of single and double surface reflections to RCS of the dihedral. Results for PO+PO double surface integral (dashed line) computed by gaussian cuadrature, and GO+PO ray-tracing (solid line) computed by the graphical processing technique (dashed line)

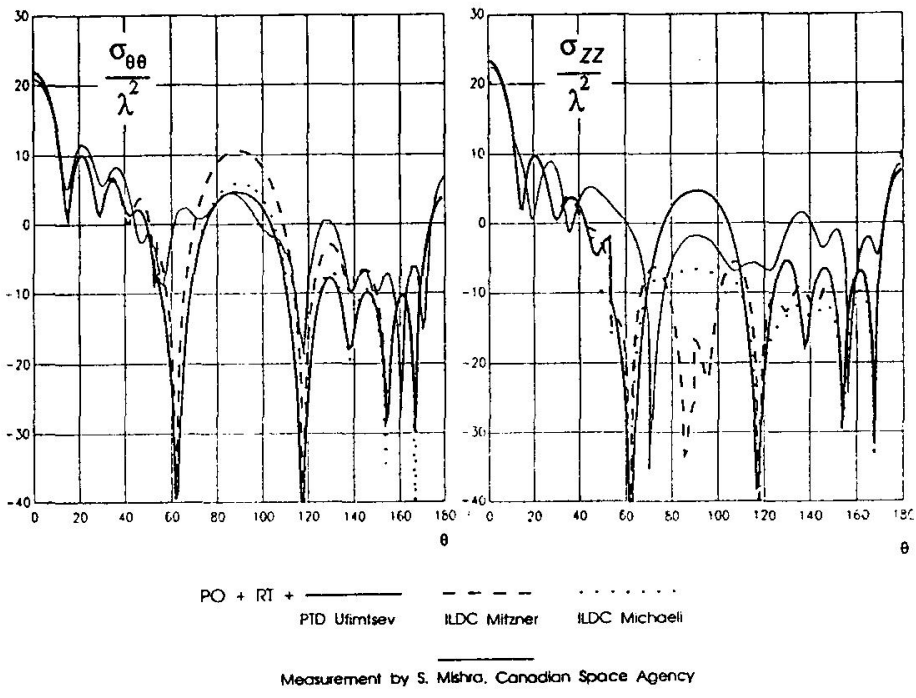

Figure 3: Results of GRECO code (solid, dashed or doted thick line) for the three kinds of edge diffraction coefficients compared with compact-range measurements (solid thin line) compared with the compact range measurements of S. Mishra, performed at the Canadian Space Agency (thin line plot).

It should be noted that there are two main sources of error in the GREC0 code analysis of this object: the electrical size is not large enough for the high frequency methods to be accurate and there exists an important contribution of surface-edge interactions that are not taken into account by GRECO.

The best results for $\theta \theta$ polarisation are obtained with Ufimtsev s PTD or Michaeli s ILDC, except for some directions of observation where Mitzner s ILDC are more accurate. In the case of $\mathrm{ZZ}$ polarisation, the behaviour of Mitzner S ILDC is worse than that of PTD or Michaeli s ILDC.

\section{Conclusions}

GRECO code has been improved by the implementation of a GOP0 graphical ray-tracing algorithm for analysis of multiple surface reflections and Michaeli s ILDC for oblique edge diffraction. The results for a $90^{\circ}$ dihedral with oblique monostatic incidence have been compared to RCS measurements. The errors due to the not large electrical size of the object and the surface-edge interactions degrade significantly the accuracy of the results. However, it can be noticed that even in these adverse conditions, GRECO is able to predict the approximate level of the RCS, to the degree of accuracy usually required when complex radar targets are analysed.

The conclusion of the comparison between the three kinds of diffraction coefficients is the following: although Mitzner s or Michaeli S ILDC are in theory more accurate than Ufimtsev s PTD for analysing oblique edges, the improvement over PTD is not significant when other unpredicted contributions to RCS are present, which is always the case for complex aircraft. Moreover, the contribution of oblique edges is very rarely significant against that of perpendicular surfaces or edges, so that the linear approximation to PTD coefficients presented in (2) and (3) is probably the most efficient approach to real-time monostatic RCS prediction.

\section{Acknowledgments}

This work has been supported by the Spanish "Comision Interministerial de Ciencia y Tecnología" (CICYT) under the project: TIC $88-288 \mathrm{E}$.

\section{References}

(1) Juan M. Rius, M. Ferrando, L. Jofre, "GREC0: Graphical Electromagnetic Computing for RCS Prediction in Real Time", IEEE Antennas and Propag. Magazine, April 1993

(2) Juan M. Rius, M. Ferrando, L. Jofre, "RCS of Complex Radar Targets in Real Time", accepted for publication in IEEE Trans. on Antennas and Propag.

(3) Juan M. Rius, "Seccion recta de blancos radar complejos en tiempo real", Doctorate Thesis Dissertation, Universitat Politècnica de Catalunya, July 1991.

(4) "SER Marseille-92, études comparatives de Surfaces Equivalentes Radar de corps infinitement conducteurs ou à impédance de surface et de cibles inhomogènes", workshop on RCS organized by Dassault-Aviation, Société Mothesim and the Universite de Provence.

(5) E.F. Knott, J.F. Shaeffer, M.T. Tuley, "Radar Cross section: Its Prediction, Measurement and Reduction", Artech House, Inc., Dedham, MA, 1985.

(6) A. Michaeli, "Elimination of Infinities in Equivalent Edge Currents, Part I: Fringe Current Components", IEEE Trans. on Antennas and Propag., Vol. AP-34, No.7, July 1986 